\title{
Analogues of Conditional Wiener Integrals with Drift and Initial Distribution on a Function Space
}

\author{
Dong Hyun Cho \\ Department of Mathematics, Kyonggi University, Suwon 443-760, Republic of Korea \\ Correspondence should be addressed to Dong Hyun Cho; j94385@kyonggi.ac.kr
}

Received 4 April 2014; Accepted 27 May 2014; Published 18 June 2014

Academic Editor: Allan Peterson

Copyright (C) 2014 Dong Hyun Cho. This is an open access article distributed under the Creative Commons Attribution License, which permits unrestricted use, distribution, and reproduction in any medium, provided the original work is properly cited.

Let $C[0, T]$ denote a generalized Wiener space, the space of real-valued continuous functions on the interval $[0, T]$, and define a stochastic process $Z: C[0, T] \times[0, T] \rightarrow \mathbb{R}$ by $Z(x, t)=\int_{0}^{t} h(u) d x(u)+x(0)+a(t)$, for $x \in C[0, T]$ and $t \in[0, T]$, where $h \in L_{2}[0, T]$ with $h \neq 0$ a.e. and $a$ is a continuous function on $[0, T]$. Let $Z_{n}: C[0, T] \rightarrow \mathbb{R}^{n+1}$ and $Z_{n+1}: C[0, T] \rightarrow \mathbb{R}^{n+2}$ be given by $Z_{n}(x)=$ $\left(Z\left(x, t_{0}\right), Z\left(x, t_{1}\right), \ldots, Z\left(x, t_{n}\right)\right)$ and $Z_{n+1}(x)=\left(Z\left(x, t_{0}\right), Z\left(x, t_{1}\right), \ldots, Z\left(x, t_{n}\right), Z\left(x, t_{n+1}\right)\right)$, where $0=t_{0}<t_{1}<\cdots<t_{n}<t_{n+1}=T$ is a partition of $[0, T]$. In this paper we derive two simple formulas for generalized conditional Wiener integrals of functions on $C[0, T]$ with the conditioning functions $Z_{n}$ and $Z_{n+1}$ which contain drift and initial distribution. As applications of these simple formulas we evaluate generalized conditional Wiener integrals of the function $\exp \left\{\int_{0}^{T} Z(x, t) d m_{L}(t)\right\}$ including the time integral on $C[0, T]$.

\section{Introduction}

A time integral is simply the Riemann integral of a function of the continuous random variable $X(x, t)=x(t)$ with respect to the parameter $t$ for $x \in C_{0}[0, T]$ which is the Wiener space, the space of continuous real-valued functions $x$ on $[0, T]$ with $x(0)=0[1]$. This means that the time integral of $X(x, t)$ is a random variable $Y$ on $C_{0}[0, T]$ satisfying

$$
Y(x)=\int_{0}^{T} F(t, X(x, t)) d m_{L}(t),
$$

where $F(t, X(x, t))$ is Riemann integrable on $[0, T]$ and $m_{L}$ is the Lebesgue measure on $\mathbb{R}$. A study of the Feynman integral provides ready examples of the utility of the time integral. The Feynman-Kac formula represents an important step in the process of providing a rigorous definition of the Feynman integral. A detailed explanation of this formula can be found in [2]. The Feynman-Kac functional is given by

$$
\exp \left\{-\int_{0}^{T} V(t, X(x, t)) d m_{L}(t)\right\}
$$

including the time integral, where $X(x, t)$ is a standard Brownian motion process on $C_{0}[0, T] \times[0, T]$ and $V$ is a complex-valued potential. Calculations involving the conditional expectations of the functional are important in the study of the Feynman integral. More examples of functionals involving time integrals are found in [2-4]. In [5] Park and Skoug introduced a generalized Brownian motion process $X: C_{0}[0, T] \times[0, T] \rightarrow \mathbb{R}$ defined by

$$
X(x, t)=\int_{0}^{t} h(u) d x(u) \quad \text { for } x \in C_{0}[0, T], t \in[0, T]
$$

where $h$ is of bounded variation with $h \neq 0$ a.e. on $[0, T]$ and the integral denotes the Paley-Wiener-Zygmund stochastic integral of $h$ according to $x$ [6], and then they generalized various theories related to the conditional Wiener integrals on $C_{0}[0, T]$.

On the other hand let $C[0, T]$ denote the space of continuous real-valued functions on the interval $[0, T]$. Im et al. [6-8] introduced a probability measure $w_{\varphi}$ on $C[0, T]$, where $\varphi$ is a probability measure on the Borel class of $\mathbb{R}$. We note that $w_{\varphi}$ is exactly the Wiener measure on $C_{0}[0, T]$ if $\varphi=\delta_{0}$, the Dirac measure concentrated at 0 . Let $a$ be 
a continuous function on $[0, T]$. Define stochastic processes $X, Y, Z: C[0, T] \times[0, T] \rightarrow \mathbb{R}$ by

$$
\begin{gathered}
X(x, t)=\int_{0}^{t} h(u) d x(u), \quad Y(x, t)=X(x, t)+a(t), \\
Z(x, t)=Y(x, t)+x(0)
\end{gathered}
$$

for $x \in C[0, T]$ and for $t \in[0, T]$. Let $Z_{n}: C[0, T] \rightarrow \mathbb{R}^{n+1}$ and $Z_{n+1}: C[0, T] \rightarrow \mathbb{R}^{n+2}$ be given by

$$
\begin{gathered}
Z_{n}(x)=\left(Z\left(x, t_{0}\right), Z\left(x, t_{1}\right), \ldots, Z\left(x, t_{n}\right)\right), \\
Z_{n+1}(x)=\left(Z\left(x, t_{0}\right), Z\left(x, t_{1}\right), \ldots, Z\left(x, t_{n}\right), Z\left(x, t_{n+1}\right)\right),
\end{gathered}
$$

where $0=t_{0}<t_{1}<\cdots<t_{n}<t_{n+1}=T$ is a partition of $[0, T]$. The author [9-11] derived various properties of $X$ and then extended his works on $X$ to $Y$. In fact he [9] established that $X$ is a generalized Brownian process and derived a simple formula for a conditional expectation which evaluates conditional expectations in terms of ordinary expectations. Moreover he [10] extended the results on $X$ to those on $Y$ with drift using only a translation theorem [6, Theorem 3.1]. In [11] he investigated the distribution of $Z$ and proved that $Z$ is a generalized Brownian motion process if the initial distribution is degenerated. He also established a generalized Wiener integration theorem which extends Lemma 2.1 of [6]. Furthermore he derived a generalized Paley-Wiener theorem which generalizes Theorem 3.5 of [6]. As applications of the theorems he evaluated generalized Wiener integrals of various functions including

$$
\begin{gathered}
Z\left(x, s_{1}\right) Z\left(x, s_{2}\right) \cdots Z\left(x, s_{m}\right), \\
\exp \left\{\int_{0}^{T} Z(x, t) d m_{L}(t)\right\}
\end{gathered}
$$

for $x \in C[0, T]$, where $0<s_{1}<s_{2}<\cdots<s_{m} \leq T$ and $m$ is a positive integer.

In this paper, using the results in [11], we derive two simple formulas for generalized conditional Wiener integrals of functions on $C[0, T]$ with the conditioning functions $Z_{n}$ and $Z_{n+1}$ which contain drift and initial distribution. As applications of these simple formulas we evaluate several generalized conditional Wiener integrals of the functions given by (6). We note that if $h \equiv 1$ and $a \equiv 0$ on $[0, T]$, then $X$ as above is exactly the stochastic process given by $X(x, t)=x(t)$ so that these works generalize the results of $[5,9,10,12-15]$ in which the works are the first results among them.

\section{Simple Formulas for Generalized Conditional Wiener Integrals}

In this section we derive two simple formulas for generalized conditional Wiener integrals on the space $\left(C[0, T], w_{\varphi}\right)$ which is introduced in the previous section.
Let $m_{L}$ denote the Lebesgue measure on the Borel class $\mathscr{B}(\mathbb{R})$ of $\mathbb{R}$ and let $\langle\cdot, \cdot\rangle_{\mathbb{R}}$ denote the dot product on $\mathbb{R}$. Let $\left(C[0, T], \mathscr{B}(C[0, T]), w_{\varphi}\right)$ be the analogue of Wiener space associated with a probability measure $\varphi$ on $\mathscr{B}(\mathbb{R})$, where $\mathscr{B}(C[0, T])$ denotes the Borel class of $C[0, T][6-8]$. Let $F$ : $C[0, T] \rightarrow \mathbb{C}$ be integrable and $X_{\tau}$ be a random vector on $C[0, T]$ assuming that the value space of $X_{\tau}$ is a normed space equipped with the Borel $\sigma$-algebra. Then we have the conditional expectation $E\left[F \mid X_{\tau}\right]$ of $F$ given $X_{\tau}$ from a well-known probability theory. Furthermore there exists a $P_{X_{\tau}}$-integrable complex valued function $\psi$ on the value space of $X_{\tau}$ such that $E\left[F \mid X_{\tau}\right](x)=\left(\psi \circ X_{\tau}\right)(x)$ for $w_{\varphi}$ a.e. $x \in C[0, T]$, where $P_{X_{\tau}}$ is the probability distribution of $X_{\tau}$. The function $\psi$ is called the conditional $w_{\varphi}$-integral of $F$ given $X_{\tau}$ and it is also denoted by $E\left[F \mid X_{\tau}\right]$.

For $v$ in $L_{2}[0, T]$ and $x$ in $C[0, T]$ let $(v, x)$ denote the Paley-Wiener-Zygmund integral of $v$ according to $x[6]$ and let $\langle\cdot, \cdot\rangle_{2}$ denote the inner product over $L_{2}[0, T]$. Let $h \in$ $L_{2}[0, T]$ be of bounded variation with $h \neq 0$ a.e. on $[0, T]$ and let $a \in C[0, T]$. Define $X_{0}(x)=x(0)$ for $x \in C[0, T]$ and define stochastic processes $X, Y, Z: C[0, T] \times[0, T] \rightarrow \mathbb{R}$ by

$$
\begin{gathered}
X(x, t)=\left(\chi_{[0, t]} h, x\right), \quad Y(x, t)=\left(\chi_{[0, t]} h, x\right)+a(t), \\
Z(x, t)=\left(\chi_{[0, t]} h, x\right)+X_{0}(x)+a(t)
\end{gathered}
$$

for $x \in C[0, T]$ and for $t \in[0, T]$. Let $b(t)=\int_{0}^{t}(h(u))^{2} d u$ and let $0=t_{0}<t_{1}<\cdots<t_{n}<t_{n+1}=T$ be a partition of $[0, T]$. For $j=1, \ldots, n+1$ let

$$
\alpha_{j}^{b}(t)=\frac{b\left(t_{j}\right)-b(t)}{b\left(t_{j}\right)-b\left(t_{j-1}\right)}, \quad \beta_{j}^{b}(t)=\frac{b(t)-b\left(t_{j-1}\right)}{b\left(t_{j}\right)-b\left(t_{j-1}\right)}
$$

for $t \in\left[t_{j-1}, t_{j}\right]$ and

$$
\sigma_{j}^{b}(s, t)=\alpha_{j}^{b}(t) \beta_{j}^{b}(s)\left(b\left(t_{j}\right)-b\left(t_{j-1}\right)\right)
$$

for $s, t \in\left[t_{j-1}, t_{j}\right]$. Define random vectors $Z_{n}: C[0, T] \rightarrow$ $\mathbb{R}^{n+1}$ and $Z_{n+1}: C[0, T] \rightarrow \mathbb{R}^{n+2}$ by

$$
\begin{gathered}
Z_{n}(x)=\left(Z\left(x, t_{0}\right), Z\left(x, t_{1}\right), \ldots, Z\left(x, t_{n}\right)\right), \\
Z_{n+1}(x)=\left(Z\left(x, t_{0}\right), Z\left(x, t_{1}\right), \ldots, Z\left(x, t_{n}\right), Z\left(x, t_{n+1}\right)\right)
\end{gathered}
$$

for $x \in C[0, T]$. For any function $f$ on $[0, T]$ define the polygonal function $P_{b, n+1}(f)$ of $f$ by

$$
\begin{aligned}
& P_{b, n+1}(f)(t) \\
& =\sum_{j=1}^{n+1} \chi_{\left(t_{j-1}, t_{j}\right]}(t)\left(\alpha_{j}^{b}(t) f\left(t_{j-1}\right)\right. \\
& \left.\quad+\beta_{j}^{b}(t) f\left(t_{j}\right)\right)+\chi_{\left\{t_{0}\right\}}(t) f\left(t_{0}\right)
\end{aligned}
$$

for $t \in[0, T]$, where $\chi_{\left(t_{j-1}, t_{j}\right]}$ and $\chi_{\left\{t_{0}\right\}}$ denote the indicator functions. For $\vec{\xi}_{n+1}=\left(\xi_{0}, \xi_{1}, \ldots, \xi_{n}, \xi_{n+1}\right) \in \mathbb{R}^{n+2}$ define 
the polygonal function $P_{b, n+1}\left(\vec{\xi}_{n+1}\right)$ of $\vec{\xi}_{n+1}$ by (11), where $f\left(t_{j}\right)$ is replaced by $\xi_{j}$, respectively, for $j=0,1, \ldots, n+1$. If $\vec{\xi}_{n}=\left(\xi_{0}\right.$, $\left.\xi_{1}, \ldots, \xi_{n}\right) \in \mathbb{R}^{n+1}, P_{b, n}\left(\vec{\xi}_{n}\right)$ is interpreted as $\chi_{\left[0, t_{n}\right]} P_{b, n+1}\left(\vec{\xi}_{n+1}\right)$ on $[0, T]$. For $x \in C[0, T]$ and for $t \in[0, T]$ let

$$
\begin{aligned}
Z_{b, n+1}(x, t) & =Z(x, t)-P_{b, n+1}(Z(x, \cdot))(t), \\
A_{t} & =a(t)-P_{b, n+1}(a)(t)
\end{aligned}
$$

and for $t \in\left[t_{j-1}, t_{j}\right]$ let

$$
h_{j, t}(u)=\left[\alpha_{j}^{b}(t) \chi_{\left[t_{j-1}, t\right]}(u)-\beta_{j}^{b}(t) \chi_{\left[t, t_{j}\right]}(u)\right] h(u)
$$

for $u \in[0, T]$. It is not difficult to show that

$$
\begin{array}{r}
Z_{b, n+1}(x, t)=\left(h_{j, t}, x\right)+A_{t} \quad \text { for } t \in\left[t_{j-1}, t_{j}\right], \\
P_{b, n+1}(Y(x, \cdot))=P_{b, n+1}(X(x, \cdot))+P_{b, n+1}(a), \\
P_{b, n+1}(Z(x, \cdot))=P_{b, n+1}(Y(x, \cdot))+X_{0}(x) \\
\text { for } x \in C[0, T] .
\end{array}
$$

Now we have the following theorem [11].

Theorem 1. For $t \in[0, T], X_{0}$ and $X(\cdot, t)$ are independent so that $X_{0}$ and $Y(\cdot, t)$ are also independent.

Theorem 2. Let $t_{j-1}<t<t_{j}$ for some $j \in\{1, \ldots, n+1\}$. Then $Z_{b, n+1}(\cdot, t)$ is normally distributed with mean $A_{t}$ and variance $\sigma_{j}^{b}(t, t)$.

Proof. By (14) and Theorem 3.4 of [6], $Z_{b, n+1}(\cdot, t)$ is normally distributed with mean $A_{t}$ and variance $\left\|h_{j, t}\right\|_{2}^{2}$. A simple calculation with an aid of (13) shows that

$$
\begin{aligned}
\left\|h_{j, t}\right\|_{2}^{2}= & \left(\left(b\left(t_{j}\right)-b(t)\right)^{2}\left(b(t)-b\left(t_{j-1}\right)\right)\right. \\
& \left.+\left(b(t)-b\left(t_{j-1}\right)\right)^{2}\left(b\left(t_{j}\right)-b(t)\right)\right) \\
& \times\left(\left(b\left(t_{j}\right)-b\left(t_{j-1}\right)\right)^{2}\right)^{-1} \\
= & \sigma_{j}^{b}(t, t)
\end{aligned}
$$

which completes the proof.

Remark 3. (1) We can prove Theorem 2 using the Fourier transform of $Z_{b, n+1}(\cdot, t)$, Lemma 2.4 of [11], and Corollaries 2.11, 2.12 of [11], but the proof is tedious.

(2) We can also prove Theorem 2 using Theorem 2.9 of [9] and Theorem 2.13 of [11].

Theorem 4. The process $\left\{Z_{b, n+1}(\cdot, t): 0 \leq t \leq T\right\}$ and $Z_{n+1}$ are stochastically independent.

Proof. Note that

$$
\begin{aligned}
Z_{b, n+1}(x, t)=X & (x, t)-P_{b, n+1}(X(x, \cdot))(t)+A_{t}, \\
Z_{n+1}(x)= & \left(X\left(x, t_{0}\right), \ldots, X\left(x, t_{n+1}\right)\right) \\
& +\left(a\left(t_{0}\right)+X_{0}(x), \ldots, a\left(t_{n+1}\right)+X_{0}(x)\right)
\end{aligned}
$$

for $x \in C[0, T] . X(x, t)-P_{b, n+1}(X(x, \cdot))(t)$ and $X\left(x, t_{j}\right)$ are independent by Theorem 2.10 of [9] for $j=1, \ldots, n+1$, and $X(\cdot, t)-P_{b, n+1}(X(\cdot, \cdot))(t)$ and $X_{0}$ are also independent by Theorem 1 which completes the proof of the theorem.

Since $Z_{b, n+1}(x, t)=Y(x, t)-P_{b, n+1}(Y(x, \cdot))(t)$ for $x \in$ $C[0, T]$ and $t \in[0, T]$, we have the following theorem by Theorem 16 of [10].

Theorem 5. The processes $\left\{Z_{b, n+1}(\cdot, t): t_{j-1} \leq t \leq t_{j}\right\}$, where $j=1, \ldots, n+1$, are stochastically independent.

For a function $F: C[0, t] \rightarrow \mathbb{C}$ let $F_{Z}(x)=F(Z(x, \cdot))$ for $x \in C[0, T]$. Applying the same method as used in the proof of Theorem 2 in [15] with Problem 4 of [16, page 216], we have the following theorem from Theorem 4.

Theorem 6. Let $F$ be a complex valued function on $C[0, T]$ and let $F_{Z}$ be integrable over $C[0, T]$. Then for a Borel subset $B$ of $\mathbb{R}^{n+2}$

$$
\begin{aligned}
& \int_{Z_{n+1}^{-1}(B)} F_{Z}(x) d w_{\varphi}(x) \\
& \quad=\int_{B} E\left[F\left(Z_{b, n+1}(x, \cdot)+P_{b, n+1}\left(\vec{\xi}_{n+1}\right)\right)\right] d P_{Z_{n+1}}\left(\vec{\xi}_{n+1}\right),
\end{aligned}
$$

where $P_{Z_{n+1}}$ is the probability distribution of $Z_{n+1}$ on $\left(\mathbb{R}^{n+2}\right.$, $\mathscr{B}\left(\mathbb{R}^{n+2}\right)$ ), so that for $P_{Z_{n+1}}$ a.e. $\vec{\xi}_{n+1} \in \mathbb{R}^{n+2}$

$$
E\left[F_{Z} \mid Z_{n+1}\right]\left(\vec{\xi}_{n+1}\right)=E\left[F\left(Z_{b, n+1}(x, \cdot)+P_{b, n+1}\left(\vec{\xi}_{n+1}\right)\right)\right],
$$

where the expectation is taken over the variable $x$.

Using similar method as used in the proof of Theorem 18 of [10], we can prove the following theorem.

Theorem 7. Let $F$ be a complex valued function on $C[0, T]$ and let $F_{Z}$ be integrable over $C[0, T]$. Let $P_{Z_{n}}$ be the probability distribution of $Z_{n}$ on $\left(\mathbb{R}^{n+1}, \mathscr{B}\left(\mathbb{R}^{n+1}\right)\right)$. Then for $P_{Z_{n}}$ a.e. $\vec{\xi}_{n}=$ $\left(\xi_{0}, \ldots, \xi_{n}\right) \in \mathbb{R}^{n+1}$

$$
\begin{aligned}
& E\left[F_{Z} \mid Z_{n}\right]\left(\vec{\xi}_{n}\right) \\
& =\left[\frac{1}{2 \pi\left(b(T)-b\left(t_{n}\right)\right)}\right]^{1 / 2} \\
& \quad \times \int_{\mathbb{R}} E\left[F\left(Z_{b, n+1}(x, \cdot)+P_{b, n+1}\left(\vec{\xi}_{n+1}\right)\right)\right] \\
& \quad \quad \times \exp \left\{-\frac{\left(\xi_{n+1}-a(T)-\left(\xi_{n}-a\left(t_{n}\right)\right)\right)^{2}}{2\left(b(T)-b\left(t_{n}\right)\right)}\right\} d m_{L}\left(\xi_{n+1}\right),
\end{aligned}
$$

where $\vec{\xi}_{n+1}=\left(\xi_{0}, \ldots, \xi_{n}, \xi_{n+1}\right)$. 
Remark 8. (1) The conditioning functions $Z_{n+1}$ and $Z_{n}$ describe the positions of paths at the times $t_{0}=0, t_{1}, \ldots$, $t_{n}, t_{n+1}=T$ (the present time). $Z_{n+1}(x)$ depends on the present position $Z(x, T)$ of the path $Z(x, \cdot)$ for $x \in C[0, T]$ while $Z_{n}$ does not. Moreover if $a(0)=0, h=\sqrt{b^{\prime}}$, and $\varphi=\delta_{0}$ which is the Dirac measure concentrated at 0 , then we can obtain the space $C_{a, b}[0, T]$ in [12] by Theorem 2.13 of [11]. Furthermore if $Z$ is replaced by the generalized Brownian motion process $x(t)$ on $C_{a, b}[0, T] \times[0, T]$ and $\varphi=\delta_{0}$, then we can also obtain Theorem 3.4 of [12] by Theorem 6 . If $a \equiv 0$ and $\varphi=\delta_{0}$, then we can obtain Theorem 3 of [5] by Theorem 6 . If $n=0$ and $\varphi=\delta_{0}$, then we can obtain Remark 2.2 of [17] by Theorem 6 . If $h=1$ a.e. and $a \equiv 0$, then we can obtain Theorem 2.9 of [13] and Theorem 2.5 of [14] by Theorems 6 and 7 , respectively. Finally if $h=1$ a.e., $\varphi=\delta_{0}$, and $a \equiv 0$, then we can obtain Theorem 2 of [15] by Theorem 6 which is among the first result expressing the conditional Wiener integrals of functions on $C_{0}[0, T]$ as ordinary Wiener integrals.

(2) Theorems 6 and 7 are not generalizations of Theorem 2.12 of [9] and Theorems 17 and 18 of [10]. In Theorems 6 and 7 the conditioning functions depend on the initial distribution $\varphi$ while the conditioning functions in $[9,10]$ do not.

\section{A Multivariate Normal Distribution}

In this section we investigate the joint distribution of $\left\{Z_{b, n+1}\left(\cdot, s_{l}\right): l=1, \ldots, m\right\}$, where $t_{j-1}<s_{1}<\cdots<s_{m}<t_{j}$ for $j=1, \ldots, n+1$. In fact we prove that the random vector as above has a multivariate normal distribution which plays a key role in the next sections.

Now we begin this section with the introduction of the following lemma.

Lemma 9. Let $t_{j-1}=s_{0}<s_{1}<\cdots<s_{m}<t_{j}$ and $h_{j, s_{l}}$ be given by (13) for $l=1, \ldots, m$. Then $\left\{h_{j, s_{l}}: l=1, \ldots, m\right\}$ is a linearly independent set in $L_{2}[0, T]$.

Proof. For $c_{1}, \ldots, c_{m} \in \mathbb{R}$ let

$$
\begin{array}{r}
\sum_{l=1}^{m} c_{l} h_{j, s_{l}}(u)=\sum_{l=1}^{m} c_{l}\left[\alpha_{j}^{b}\left(s_{l}\right) \chi_{\left[t_{j-1}, s_{l}\right]}(u)\right. \\
\left.-\beta_{j}^{b}\left(s_{l}\right) \chi_{\left[s_{l}, t_{j}\right]}(u)\right] h(u)
\end{array}
$$

$$
=0
$$

for a.e. $u \in[0, T]$. For $k=1, \ldots, m$ take $u_{k} \in\left(s_{k-1}, s_{k}\right)$ which satisfies the above equation and $h\left(u_{k}\right) \neq 0$. Replacing $u$ by $u_{k}$ we have the following linear equation system with unknowns $c_{1}, \ldots, c_{m}$ :

$$
\begin{gathered}
\alpha_{j}^{b}\left(s_{1}\right) c_{1}+\alpha_{j}^{b}\left(s_{2}\right) c_{2}+\cdots+\alpha_{j}^{b}\left(s_{m-2}\right) c_{m-2} \\
+\alpha_{j}^{b}\left(s_{m-1}\right) c_{m-1}+\alpha_{j}^{b}\left(s_{m}\right) c_{m}=0
\end{gathered}
$$

$$
\begin{gathered}
-\beta_{j}^{b}\left(s_{1}\right) c_{1}+\alpha_{j}^{b}\left(s_{2}\right) c_{2}+\cdots+\alpha_{j}^{b}\left(s_{m-2}\right) c_{m-2} \\
+\alpha_{j}^{b}\left(s_{m-1}\right) c_{m-1}+\alpha_{j}^{b}\left(s_{m}\right) c_{m}=0 \\
\vdots \\
-\beta_{j}^{b}\left(s_{1}\right) c_{1}-\beta_{j}^{b}\left(s_{2}\right) c_{2}-\cdots-\beta_{j}^{b}\left(s_{m-2}\right) c_{m-2} \\
+\alpha_{j}^{b}\left(s_{m-1}\right) c_{m-1}+\alpha_{j}^{b}\left(s_{m}\right) c_{m}=0, \\
-\beta_{j}^{b}\left(s_{1}\right) c_{1}-\beta_{j}^{b}\left(s_{2}\right) c_{2}-\cdots-\beta_{j}^{b}\left(s_{m-2}\right) c_{m-2} \\
-\beta_{j}^{b}\left(s_{m-1}\right) c_{m-1}+\alpha_{j}^{b}\left(s_{m}\right) c_{m}=0 .
\end{gathered}
$$

The determinant of coefficient matrix of the system is given by

$$
\begin{array}{|}
\left|\begin{array}{cccccc}
\alpha_{j}^{b}\left(s_{1}\right) & \alpha_{j}^{b}\left(s_{2}\right) & \cdots & \alpha_{j}^{b}\left(s_{m-2}\right) & \alpha_{j}^{b}\left(s_{m-1}\right) & \alpha_{j}^{b}\left(s_{m}\right) \\
-\beta_{j}^{b}\left(s_{1}\right) & \alpha_{j}^{b}\left(s_{2}\right) & \cdots & \alpha_{j}^{b}\left(s_{m-2}\right) & \alpha_{j}^{b}\left(s_{m-1}\right) & \alpha_{j}^{b}\left(s_{m}\right) \\
\vdots & \vdots & \vdots & \vdots & \vdots & \vdots \\
-\beta_{j}^{b}\left(s_{1}\right) & -\beta_{j}^{b}\left(s_{2}\right) & \cdots & -\beta_{j}^{b}\left(s_{m-2}\right) & \alpha_{j}^{b}\left(s_{m-1}\right) & \alpha_{j}^{b}\left(s_{m}\right) \\
-\beta_{j}^{b}\left(s_{1}\right) & -\beta_{j}^{b}\left(s_{2}\right) & \cdots & -\beta_{j}^{b}\left(s_{m-2}\right) & -\beta_{j}^{b}\left(s_{m-1}\right) & \alpha_{j}^{b}\left(s_{m}\right)
\end{array}\right| \\
=\left|\begin{array}{cccccc}
\alpha_{j}^{b}\left(s_{1}\right) & \alpha_{j}^{b}\left(s_{2}\right) & \cdots & \alpha_{j}^{b}\left(s_{m-2}\right) & \alpha_{j}^{b}\left(s_{m-1}\right) & \alpha_{j}^{b}\left(s_{m}\right) \\
-1 & 0 & \cdots & 0 & 0 & 0 \\
-1 & -1 & \cdots & 0 & 0 & 0 \\
\vdots & \vdots & \vdots & \vdots & \vdots & \vdots \\
-1 & -1 & \cdots & -1 & 0 & 0 \\
-1 & -1 & \cdots & -1 & -1 & 0
\end{array}\right| \\
=(-1)^{1+m} \alpha_{j}^{b}\left(s_{m}\right)(-1)^{m-1}=\alpha_{j}^{b}\left(s_{m}\right) \neq 0 .
\end{array}
$$

Now we have $c_{1}=c_{2}=\cdots=c_{m}=0$ which completes the proof.

A random variable $X_{\tau}$ on $C[0, t]$ is said to be degenerated if there exists a constant $c$ satisfying $w_{\varphi}\left(X_{\tau}=c\right)=1$ [18].

Lemma 10. Let the assumptions and notations be as given in Lemma 9. If $c_{1}, \ldots, c_{m} \in \mathbb{R}$ and $\sum_{l=1}^{m} c_{l} Z_{b, n+1}\left(\cdot, s_{l}\right)$ is degenerated, then $c_{l}=0$ for all $l=1, \ldots, m$.

Proof. By (14) we can take a real constant $c$ satisfying

$$
c=\sum_{l=1}^{m} c_{l} Z_{b, n+1}\left(x, s_{l}\right)=\left(\sum_{l=1}^{m} c_{l} h_{j, s_{l}}, x\right)+\sum_{l=1}^{m} c_{l} A_{s_{l}}
$$

for a.e. $x \in C[0, T]$. Then we have by Theorem 3.4 of [6] that

$$
\begin{aligned}
0 & =\int_{C[0, T]}\left[\left(\sum_{l=1}^{m} c_{l} h_{j, s_{l}}, x\right)+\sum_{l=1}^{m} c_{l} A_{s_{l}}-c\right]^{2} d w_{\varphi}(x) \\
& =\left\|\sum_{l=1}^{m} c_{l} h_{j, s_{l}}\right\|_{2}^{2}+\left[\sum_{l=1}^{m} c_{l} A_{s_{l}}-c\right]^{2}
\end{aligned}
$$


so that $\sum_{l=1}^{m} c_{l} h_{j, s_{l}}=0$ in $L_{2}[0, T]$. Now $c_{1}=c_{2}=\cdots=c_{m}=0$ by Lemma 9 which completes the proof.

Lemma 11. Let the assumptions and notations be as given in Lemma 9. Then for $\vec{s}=\left(s_{1}, \ldots, s_{m}\right)$ the covariance matrix $\Sigma_{m}(\vec{s})=\left[\sigma\left(s_{l}, s_{k}\right)\right]_{m \times m}$ of the random variables $Z_{b, n+1}\left(\cdot, s_{l}\right)$, $l=1, \ldots, m$, exists and is positive definite. Moreover $\sigma\left(s_{l}, s_{k}\right)$ is given by

$$
\sigma\left(s_{l}, s_{k}\right)=\left\langle h_{j, s_{l}}, h_{j, s_{k}}\right\rangle_{2}=\sigma_{j}^{b}\left(\min \left\{s_{l}, s_{k}\right\}, \max \left\{s_{l}, s_{k}\right\}\right)
$$

and the determinant $\left|\Sigma_{m}(\vec{s})\right|$ is positive so that $\Sigma_{m}(\vec{s})$ is nonsingular and the inverse matrix $\Sigma_{m}^{-1}(\vec{s})$ of $\Sigma_{m}(\vec{s})$ is also positive definite.

Proof. By Theorem 3.4 of [6]

$$
\begin{aligned}
& \left\|h_{j, s_{l}}\right\|_{2}^{2}+2 \int_{C[0, T]}\left(h_{j, s_{l}}, x\right)\left(h_{j, s_{k}}, x\right) d w_{\varphi}(x)+\left\|h_{j, s_{k}}\right\|_{2}^{2} \\
& =\int_{C[0, T]}\left(h_{j, s_{l}}+h_{j, s_{k}}, x\right)^{2} d w_{\varphi}(x) \\
& \quad=\left\|h_{j, s_{l}}\right\|_{2}^{2}+2\left\langle h_{j, s_{l}}, h_{j, s_{k}}\right\rangle_{2}+\left\|h_{j, s_{k}}\right\|_{2}^{2}
\end{aligned}
$$

so that the covariance $\sigma\left(s_{l}, s_{k}\right)$ of $Z_{b, n+1}\left(\cdot, s_{l}\right)$ and $Z_{b, n+1}\left(\cdot, s_{k}\right)$ is given by $\sigma\left(s_{l}, s_{k}\right)=\left\langle h_{j, s_{l}}, h_{j, s_{k}}\right\rangle_{2}$. Now we have for $l \leq k$

$$
\begin{aligned}
\sigma\left(s_{l}, s_{k}\right)= & \int_{0}^{T}\left[\alpha_{j}^{b}\left(s_{l}\right) \chi_{\left[t_{j-1}, s_{l}\right]}(u)-\beta_{j}^{b}\left(s_{l}\right) \chi_{\left[s_{l}, t_{j}\right]}(u)\right] \\
& \times\left[\alpha_{j}^{b}\left(s_{k}\right) \chi_{\left[t_{j-1}, s_{k}\right]}(u)-\beta_{j}^{b}\left(s_{k}\right) \chi_{\left[s_{k}, t_{j}\right]}(u)\right] \\
& \times(h(u))^{2} d m_{L}(u) \\
= & \alpha_{j}^{b}\left(s_{l}\right) \alpha_{j}^{b}\left(s_{k}\right)\left(b\left(s_{l}\right)-b\left(t_{j-1}\right)\right) \\
& -\beta_{j}^{b}\left(s_{l}\right) \alpha_{j}^{b}\left(s_{k}\right)\left(b\left(s_{k}\right)-b\left(s_{l}\right)\right) \\
& +\beta_{j}^{b}\left(s_{l}\right) \beta_{j}^{b}\left(s_{k}\right)\left(b\left(t_{j}\right)-b\left(s_{k}\right)\right) \\
= & \frac{\left(b\left(t_{j}\right)-b\left(s_{k}\right)\right)\left(b\left(s_{l}\right)-b\left(t_{j-1}\right)\right)}{b\left(t_{j}\right)-b\left(t_{j-1}\right)} \\
= & \sigma_{j}^{b}\left(s_{l}, s_{k}\right)
\end{aligned}
$$

which proves (26). We have for $\vec{c}=\left(c_{1}, \ldots, c_{m}\right) \in \mathbb{R}^{m}$

$$
\begin{aligned}
& \left\langle\Sigma_{m}(\vec{s}) \vec{c}, \vec{c}\right\rangle_{\mathbb{R}} \\
& =\sum_{l=1}^{m} \sum_{k=1}^{m} \sigma\left(s_{l}, s_{k}\right) c_{l} c_{k} \\
& =E\left[\left(\sum_{l=1}^{m} c_{l}\left(Z_{b, n+1}\left(\cdot, s_{l}\right)-A_{s_{l}}\right)\right)^{2}\right] \geq 0 .
\end{aligned}
$$

Moreover if $\left\langle\Sigma_{m}(\vec{s}) \vec{c}, \vec{c}\right\rangle_{\mathbb{R}}=0$, then

$$
\sum_{l=1}^{m} c_{l} Z_{b, n+1}\left(x, s_{l}\right)=\sum_{l=1}^{m} c_{l} A_{s_{l}}
$$

for a.e. $x \in C[0, T]$; that is, $\sum_{l=1}^{m} c_{l} Z_{b, n+1}\left(\cdot, s_{l}\right)$ is degenerated. Thus the covariance matrix $\Sigma_{m}(\vec{s})$ is positive definite by Lemma 10. Since $\Sigma_{m}(\vec{s})$ is symmetric and positive definite, the eigenvalues $\lambda_{1}, \ldots, \lambda_{m}$ of $\Sigma_{m}(\vec{s})$ are real and positive. Since $\left|\Sigma_{m}(\vec{s})\right|=\lambda_{1} \cdots \lambda_{m}$, we have $\left|\Sigma_{m}(\vec{s})\right|>0$ so that $\Sigma_{m}(\vec{s})$ is invertible. Since

$$
\begin{aligned}
\left\langle\Sigma_{m}^{-1}(\vec{s}) \vec{c}, \vec{c}\right\rangle_{\mathbb{R}} & =\left\langle\vec{c}, \Sigma_{m}^{-1}(\vec{s}) \vec{c}\right\rangle_{\mathbb{R}} \\
& =\left\langle\Sigma_{m}(\vec{s}) \Sigma_{m}^{-1}(\vec{s}) \vec{c}, \Sigma_{m}^{-1}(\vec{s}) \vec{c}\right\rangle_{\mathbb{R}} \geq 0
\end{aligned}
$$

and $\left\langle\Sigma_{m}^{-1}(\vec{s}) \vec{c}, \vec{c}\right\rangle_{\mathbb{R}}=0$ implies $\Sigma_{m}^{-1}(\vec{s}) \vec{c}=\overrightarrow{0}$; that is, $\vec{c}=\overrightarrow{0}$, $\Sigma_{m}^{-1}(\vec{s})$ is positive definite.

Remark 12. Using the same process as used in the proof of Theorem 3.4 in [9] we can prove (26).

For simplicity let

$$
\begin{aligned}
\Psi_{m}(\vec{s}, \vec{u})= & {\left[\frac{1}{(2 \pi)^{m}\left|\Sigma_{m}(\vec{s})\right|}\right]^{1 / 2} } \\
& \times \exp \left\{-\frac{1}{2}\left\langle\Sigma_{m}^{-1}(\vec{s})(\vec{u}-A(\vec{s})), \vec{u}-A(\vec{s})\right\rangle_{\mathbb{R}}\right\}
\end{aligned}
$$

for $\vec{u} \in \mathbb{R}^{m}$ and $\vec{s}=\left(s_{1}, \ldots, s_{m}\right) \in \mathbb{R}^{m}$, where $t_{j-1}<s_{1}<\cdots<$ $s_{m}<t_{j}$ and $A(\vec{s})=\left(A_{s_{1}}, \ldots, A_{s_{m}}\right)$. By Lemmas $9,10,11$, and Theorem 4 of [1] we have the following theorem which is our main result in this section.

Theorem 13. Let the assumptions and notations be as given in Lemma 11. Then for every Borel measurable function $f$ : $\mathbb{R}^{m} \rightarrow \mathbb{C}$

$$
\begin{gathered}
\int_{\mathbb{C}[0, T]} f\left(Z_{b, n+1}\left(x, s_{1}\right), \ldots, Z_{b, n+1}\left(x, s_{m}\right)\right) d w_{\varphi}(x) \\
\stackrel{*}{=} \int_{\mathbb{R}^{m}} f(\vec{u}) \Psi_{m}(\vec{s}, \vec{u}) d\left(m_{L}\right)^{m}(\vec{u}) \\
=\left[\frac{1}{(2 \pi)^{m}}\right]^{1 / 2} \int_{\mathbb{R}^{m}} f\left(\sqrt{\sum_{m}(\vec{s})} \vec{u}+A(\vec{s})\right) \\
\times \exp \left\{-\frac{1}{2}\|\vec{u}\|_{\mathbb{R}}^{2}\right\} d\left(m_{L}\right)^{m}(\vec{u}),
\end{gathered}
$$

where $\sqrt{\Sigma_{m}(\vec{s})}$ is the positive definite matrix satisfying ${\sqrt{\Sigma_{m}(\vec{s})}}^{2}=\Sigma_{m}(\vec{s})$ and $\stackrel{*}{=}$ means that if either side exists, then both sides exist and they are equal. 


\section{Conditional Expectations of Functions on Time Integrals}

In this section we evaluate generalized conditional Wiener integrals of the function $\exp \left\{\int_{0}^{T} Z(x, t) d m_{L}(t)\right\}$ including a time integral. To do this we have the following theorem by Theorems 6 and 13 and Theorem 3.3 of [11].

Theorem 14. Let the assumptions and notations be as given in Theorem 13 and let $H_{m}(\vec{s}, x)=x\left(s_{1}\right) \cdots x\left(s_{m}\right)$ for $x \in C[0, T]$. Suppose that $\int_{\mathbb{R}}|u|^{m} d \varphi(u)<\infty$. Then for $P_{Z_{n+1}}$ a.e. $\vec{\xi}_{n+1} \in$ $\mathbb{R}^{n+2}$

$$
\begin{aligned}
E & {\left[\left(H_{m}(\vec{s}, \cdot)\right)_{Z} \mid Z_{n+1}\right]\left(\vec{\xi}_{n+1}\right) } \\
& =\int_{\mathbb{R}^{m}}\left(u_{1}+P_{b, n+1}\left(\vec{\xi}_{n+1}\right)\left(s_{1}\right)\right)
\end{aligned}
$$

where $\vec{u}=\left(u_{1}, \ldots, u_{m}\right)$.

Example 15. Let the assumptions and notations be as given in Theorem 14. If $m=1$, then by Theorem 2 we have for $P_{Z_{n+1}}$ a.e. $\vec{\xi}_{n+1} \in \mathbb{R}^{n+2}$

$$
E\left[\left(H_{1}\left(s_{1}, \cdot\right)\right)_{Z} \mid Z_{n+1}\right]\left(\vec{\xi}_{n+1}\right)=A_{s_{1}}+P_{b, n+1}\left(\vec{\xi}_{n+1}\right)\left(s_{1}\right) .
$$

If $m=2$, then

$$
\begin{gathered}
\left|\Sigma_{2}(\vec{s})\right|=\left|\begin{array}{ll}
\sigma_{j}^{b}\left(s_{1}, s_{1}\right) & \sigma_{j}^{b}\left(s_{1}, s_{2}\right) \\
\sigma_{j}^{b}\left(s_{1}, s_{2}\right) & \sigma_{j}^{b}\left(s_{2}, s_{2}\right)
\end{array}\right|=\frac{\left(b\left(t_{j}\right)-b\left(s_{2}\right)\right)\left(b\left(s_{2}\right)-b\left(s_{1}\right)\right)\left(b\left(s_{1}\right)-b\left(t_{j-1}\right)\right)}{b\left(t_{j}\right)-b\left(t_{j-1}\right)}, \\
\Sigma_{2}^{-1}(\vec{s})=\left[\begin{array}{cc}
\frac{b\left(s_{2}\right)-b\left(t_{j-1}\right)}{\left(b\left(s_{2}\right)-b\left(s_{1}\right)\right)\left(b\left(s_{1}\right)-b\left(t_{j-1}\right)\right)} & -\frac{b\left(s_{1}\right)-b\left(t_{j-1}\right)}{\left(b\left(s_{2}\right)-b\left(s_{1}\right)\right)\left(b\left(s_{1}\right)-b\left(t_{j-1}\right)\right)} \\
-\frac{b\left(s_{1}\right)-b\left(t_{j-1}\right)}{\left(b\left(s_{2}\right)-b\left(s_{1}\right)\right)\left(b\left(s_{1}\right)-b\left(t_{j-1}\right)\right)} & \frac{b\left(t_{j}\right)-b\left(s_{1}\right)}{\left(b\left(t_{j}\right)-b\left(s_{2}\right)\right)\left(b\left(s_{2}\right)-b\left(s_{1}\right)\right)}
\end{array}\right] .
\end{gathered}
$$

Moreover for $P_{Z_{n+1}}$ a.e. $\vec{\xi}_{n+1} \in \mathbb{R}^{n+2}$

$$
\begin{aligned}
E[ & \left.\left(H_{2}(\vec{s}, \cdot)\right)_{Z} \mid Z_{n+1}\right]\left(\vec{\xi}_{n+1}\right) \\
= & \int_{\mathbb{R}^{2}}\left(u_{1}+P_{b, n+1}\left(\vec{\xi}_{n+1}\right)\left(s_{1}\right)\right)\left(u_{2}+P_{b, n+1}\left(\vec{\xi}_{n+1}\right)\left(s_{2}\right)\right) \\
& \quad \times \Psi_{2}(\vec{s}, \vec{u}) d\left(m_{L}\right)^{2}(\vec{u}) \\
= & \sigma_{j}^{b}\left(s_{1}, s_{2}\right)+\left(A_{s_{1}}+P_{b, n+1}\left(\vec{\xi}_{n+1}\right)\left(s_{1}\right)\right) \\
& \times\left(A_{s_{2}}+P_{b, n+1}\left(\vec{\xi}_{n+1}\right)\left(s_{2}\right)\right)
\end{aligned}
$$

which is a generalization of (2) in Theorem 23 of [10].

By Theorem 7 we have the following theorem.

Theorem 16. Let the assumptions and notations be as given in Theorem 14 and let $j \in\{1, \ldots, n-1\}$. Then for $P_{Z_{n}}$ a.e. $\vec{\xi}_{n}=$ $\left(\xi_{0}, \xi_{1}, \ldots, \xi_{n}\right) \in \mathbb{R}^{n+1}$

$$
\begin{aligned}
& E\left[\left(H_{m}(\vec{s}, \cdot)\right)_{Z} \mid Z_{n}\right]\left(\vec{\xi}_{n}\right) \\
& =\int_{\mathbb{R}^{m}}\left(u_{1}+P_{b, n}\left(\vec{\xi}_{n}\right)\left(s_{1}\right)\right) \cdots\left(u_{m}+P_{b, n}\left(\vec{\xi}_{n}\right)\left(s_{m}\right)\right) \\
& \quad \times \Psi_{m}(\vec{s}, \vec{u}) d\left(m_{L}\right)^{m}(\vec{u}) .
\end{aligned}
$$

Example 17. Let the assumptions and notations be as given in Theorem 16. If $m=1$, then by Theorem 16 we have for $P_{Z_{n}}$ a.e. $\vec{\xi}_{n} \in \mathbb{R}^{n+1}$

$$
E\left[\left(H_{1}\left(s_{1}, \cdot\right)\right)_{Z} \mid Z_{n}\right]\left(\vec{\xi}_{n}\right)=A_{s_{1}}+P_{b, n}\left(\vec{\xi}_{n}\right)\left(s_{1}\right)
$$

If $m=2$, then we have for $P_{Z_{n}}$ a.e. $\vec{\xi}_{n} \in \mathbb{R}^{n+1}$

$$
\begin{aligned}
E\left[\left(H_{2}(\vec{s}, \cdot)\right)_{Z} \mid Z_{n}\right]\left(\vec{\xi}_{n}\right) \\
=\sigma_{j}^{b}\left(s_{1}, s_{2}\right)+\left(A_{s_{1}}+P_{b, n}\left(\vec{\xi}_{n}\right)\left(s_{1}\right)\right) \\
\quad \times\left(A_{s_{2}}+P_{b, n}\left(\vec{\xi}_{n}\right)\left(s_{2}\right)\right)
\end{aligned}
$$

which is a generalization of (2) in Theorem 24 of [10].

Theorem 18. Let the assumptions and notations be as given in Theorem 14 and let $t_{n}<s_{1}<\cdots<s_{m}<t_{n+1}=T$. For $\xi_{n} \in \mathbb{R}$ and $\vec{u}=\left(u_{1}, \ldots, u_{m}\right) \in \mathbb{R}^{m}$ let

$$
\begin{aligned}
P_{\vec{s}, \vec{u}, \xi_{n}}(z)= & \left(u_{1}+\xi_{n}+\frac{b\left(s_{1}\right)-b\left(t_{n}\right)}{b(T)-b\left(t_{n}\right)}\right. \\
& \left.\times\left(a(T)-a\left(t_{n}\right)\right)+\frac{b\left(s_{1}\right)-b\left(t_{n}\right)}{\sqrt{b(T)-b\left(t_{n}\right)}} z\right)
\end{aligned}
$$




$$
\begin{aligned}
& \ldots\left(u_{m}+\xi_{n}+\frac{b\left(s_{m}\right)-b\left(t_{n}\right)}{b(T)-b\left(t_{n}\right)}\right. \\
& \left.\quad \times\left(a(T)-a\left(t_{n}\right)\right)+\frac{b\left(s_{m}\right)-b\left(t_{n}\right)}{\sqrt{b(T)-b\left(t_{n}\right)}} z\right) \\
& =\sum_{l=0}^{m} a_{l}\left(\vec{s}, \vec{u}, \xi_{n}\right) z^{l}
\end{aligned}
$$

for $z \in \mathbb{R}$, where $\vec{s}=\left(s_{1}, \ldots, s_{m}\right)$. Then for $P_{Z_{n}}$ a.e. $\vec{\xi}_{n}=\left(\xi_{0}, \xi_{1}\right.$, $\left.\ldots, \xi_{n}\right) \in \mathbb{R}^{n+1}$

$$
\begin{aligned}
& E\left[\left(H_{m}(\vec{s}, \cdot)\right)_{Z} \mid Z_{n}\right]\left(\vec{\xi}_{n}\right) \\
&=\sum_{l=0}^{[m / 2]} \frac{(2 l) !}{2^{l} l !} \int_{\mathbb{R}^{m}} a_{2 l}\left(\vec{s}, \vec{u}, \xi_{n}\right) \\
& \quad \times \Psi_{m}(\vec{s}, \vec{u}) d\left(m_{L}\right)^{m}(\vec{u}),
\end{aligned}
$$

where $[\mathrm{m} / 2]$ denotes the greatest integer less than or equal to $m / 2$.

Proof. For $\vec{\xi}_{n}=\left(\xi_{0}, \xi_{1}, \ldots, \xi_{n}\right) \in \mathbb{R}^{n+1}$ let $\vec{\xi}_{n+1}=\left(\xi_{0}, \xi_{1}, \ldots\right.$, $\left.\xi_{n}, \xi_{n+1}\right)$, where $\xi_{n+1} \in \mathbb{R}$. By Theorems 7 and 14 we have for $P_{Z_{n}}$ a.e. $\vec{\xi}_{n} \in \mathbb{R}^{n+1}$

$$
\begin{aligned}
& E\left[\left(H_{m}(\vec{s}, \cdot)\right)_{Z} \mid Z_{n}\right]\left(\vec{\xi}_{n}\right) \\
& =\left[\frac{1}{2 \pi\left(b(T)-b\left(t_{n}\right)\right)}\right]^{1 / 2} \\
& \quad \times \int_{\mathbb{R}^{m}} \Psi_{m}(\vec{s}, \vec{u}) \\
& \quad \times \int_{\mathbb{R}}\left(u_{1}+P_{b, n+1}\left(\vec{\xi}_{n+1}\right)\left(s_{1}\right)\right) \\
& \quad \cdots\left(u_{m}+P_{b, n+1}\left(\vec{\xi}_{n+1}\right)\left(s_{m}\right)\right) \\
& \quad \times \exp \left\{-\frac{\left(\xi_{n+1}-a(T)-\left(\xi_{n}-a\left(t_{n}\right)\right)\right)^{2}}{2\left(b(T)-b\left(t_{n}\right)\right)}\right\} d m_{L}\left(\xi_{n+1}\right) d\left(m_{L}\right)^{m}(\vec{u}) \\
& =\left[\frac{1}{2 \pi\left(b(T)-b\left(t_{n}\right)\right)}\right]^{1 / 2} \\
& \quad \times \int_{\mathbb{R}^{m}} \Psi_{m}(\vec{s}, \vec{u}) \\
& \quad \times \int_{\mathbb{R}}\left(u_{1}+\xi_{n}+\frac{b\left(s_{1}\right)-b\left(t_{n}\right)}{b(T)-b\left(t_{n}\right)}\right. \\
& \left.\quad \times\left(u+a(T)-a\left(t_{n}\right)\right)\right) \\
& \quad \ldots\left(u_{m}+\xi_{n}+\frac{b\left(s_{m}\right)-b\left(t_{n}\right)}{b(T)-b\left(t_{n}\right)}\right. \\
& \left.\quad \times\left(u+a(T)-a\left(t_{n}\right)\right)\right) \\
& \quad \times \exp \left\{-\frac{u^{2}}{2\left(b(T)-b\left(t_{n}\right)\right)}\right\} d m_{L}(u) d\left(m_{L}\right)^{m}(\vec{u}) .
\end{aligned}
$$

Let $z=u / \sqrt{b(T)-b\left(t_{n}\right)}$. Then by the change of variable theorem

$$
\begin{aligned}
& E\left[\left(H_{m}(\vec{s}, \cdot)\right)_{Z} \mid Z_{n}\right]\left(\vec{\xi}_{n}\right) \\
& =\left(\frac{1}{2 \pi}\right)^{1 / 2} \int_{\mathbb{R}^{m}} \Psi_{m}(\vec{s}, \vec{u}) \\
& \quad \quad \quad \int_{\mathbb{R}} P_{\vec{s}, \vec{u}, \xi_{n}}(z) \exp \left\{-\frac{z^{2}}{2}\right\} d m_{L}(z) d\left(m_{L}\right)^{m}(\vec{u}) \\
& =\sum_{l=0}^{[m / 2]}\left(\frac{1}{2 \pi}\right)^{1 / 2} \int_{\mathbb{R}^{m}} \Psi_{m}(\vec{s}, \vec{u}) a_{2 l}\left(\vec{s}, \vec{u}, \xi_{n}\right) \\
& \quad \times \int_{\mathbb{R}} z^{2 l} \exp \left\{-\frac{z^{2}}{2}\right\} d m_{L}(z) d\left(m_{L}\right)^{m}(\vec{u}) \\
& =\sum_{l=0}^{[m / 2]} \frac{(2 l) !}{2^{l} l !} \int_{\mathbb{R}^{m}} a_{2 l}\left(\vec{s}, \vec{u}, \xi_{n}\right) \Psi_{m}(\vec{s}, \vec{u}) d\left(m_{L}\right)^{m}(\vec{u})
\end{aligned}
$$

which completes the proof.

Example 19. Let the assumptions and notations be as given in Theorem 18. If $m=1$, then by Theorem 18 we have for $P_{Z_{n}}$ a.e. $\vec{\xi}_{n}=\left(\xi_{0}, \xi_{1}, \ldots, \xi_{n}\right) \in \mathbb{R}^{n+1}$

$$
\begin{aligned}
& E\left[\left(H_{1}\left(s_{1}, \cdot\right)\right)_{Z} \mid Z_{n}\right]\left(\vec{\xi}_{n}\right) \\
& =\left[\frac{1}{2 \pi \sigma_{n+1}^{b}\left(s_{1}, s_{1}\right)}\right]^{1 / 2} \\
& \quad \times \int_{\mathbb{R}}\left(u_{1}+\xi_{n}+\frac{b\left(s_{1}\right)-b\left(t_{n}\right)}{b(T)-b\left(t_{n}\right)}\right. \\
& \left.\times\left(a(T)-a\left(t_{n}\right)\right)\right) \\
& \quad \times \exp \left\{-\frac{\left(u_{1}-A_{s_{1}}\right)^{2}}{2 \sigma_{n+1}^{b}\left(s_{1}, s_{1}\right)}\right\} d m_{L}\left(u_{1}\right) \\
& =a\left(s_{1}\right)-a\left(t_{n}\right)+\xi_{n} .
\end{aligned}
$$

If $m=2$, then we have for $P_{Z_{n}}$ a.e. $\vec{\xi}_{n}=\left(\xi_{0}, \xi_{1}, \ldots, \xi_{n}\right) \in \mathbb{R}^{n+1}$

$$
\begin{aligned}
& E[\left.\left(H_{2}(\vec{s}, \cdot)\right)_{Z} \mid Z_{n}\right]\left(\vec{\xi}_{n}\right) \\
&=\sum_{l=0}^{1} \frac{(2 l) !}{2 l l !} \int_{\mathbb{R}^{2}} a_{2 l}\left(\vec{s}, \vec{u}, \xi_{n}\right) \Psi_{2}(\vec{s}, \vec{u}) d\left(m_{L}\right)^{2}(\vec{u}) \\
&=\int_{\mathbb{R}^{2}}\left(u_{1}+\xi_{n}+\frac{b\left(s_{1}\right)-b\left(t_{n}\right)}{b(T)-b\left(t_{n}\right)}\left(a(T)-a\left(t_{n}\right)\right)\right) \\
& \quad \times\left(u_{2}+\xi_{n}+\frac{b\left(s_{2}\right)-b\left(t_{n}\right)}{b(T)-b\left(t_{n}\right)}\right. \\
&\left.\quad \times\left(a(T)-a\left(t_{n}\right)\right)\right) \Psi_{2}(\vec{s}, \vec{u}) d\left(m_{L}\right)^{2}(\vec{u}) \\
&+\frac{\left(b\left(s_{1}\right)-b\left(t_{n}\right)\right)\left(b\left(s_{2}\right)-b\left(t_{n}\right)\right)}{b(T)-b\left(t_{n}\right)}
\end{aligned}
$$




$$
\begin{aligned}
= & \sigma_{n+1}^{b}\left(s_{1}, s_{2}\right)+A_{s_{1}} A_{s_{2}}+A_{s_{1}} \\
& \times\left(\xi_{n}+\frac{b\left(s_{2}\right)-b\left(t_{n}\right)}{b(T)-b\left(t_{n}\right)}\left(a(T)-a\left(t_{n}\right)\right)\right) \\
& +A_{s_{2}}\left(\xi_{n}+\frac{b\left(s_{1}\right)-b\left(t_{n}\right)}{b(T)-b\left(t_{n}\right)}\left(a(T)-a\left(t_{n}\right)\right)\right) \\
& +\left(\xi_{n}+\frac{b\left(s_{2}\right)-b\left(t_{n}\right)}{b(T)-b\left(t_{n}\right)}\left(a(T)-a\left(t_{n}\right)\right)\right) \\
& \times\left(\xi_{n}+\frac{b\left(s_{1}\right)-b\left(t_{n}\right)}{b(T)-b\left(t_{n}\right)}\left(a(T)-a\left(t_{n}\right)\right)\right) \\
& +\frac{\left(b\left(s_{1}\right)-b\left(t_{n}\right)\right)\left(b\left(s_{2}\right)-b\left(t_{n}\right)\right)}{b(T)-b\left(t_{n}\right)} \\
= & \xi_{n}^{2}+\xi_{n}\left(A_{s_{1}}+A_{s_{2}}+\left(a(T)-a\left(t_{n}\right)\right)\right. \\
& \left.\times\left(\beta_{n+1}^{b}\left(s_{1}\right)+\beta_{n+1}^{b}\left(s_{2}\right)\right)\right) \\
& +\left(A_{s_{1}}+\beta_{n+1}^{b}\left(s_{1}\right)\left(a(T)-a\left(t_{n}\right)\right)\right) \\
& +\left(A_{s_{2}}+\beta_{n+1}^{b}\left(s_{2}\right)\left(a(T)-a\left(t_{n}\right)\right)\right) \\
& +\sigma_{n+1}^{b}\left(s_{1}\right) \beta_{n+1}^{b}\left(s_{1}, s_{2}\right)
\end{aligned}
$$

which is a generalization of (5) in Theorem 24 of [10].

Theorem 20. Let the notations be as given in Theorem 14 and let $H(x)=\exp \left\{\int_{0}^{T} x(t) d m_{L}(t)\right\}$ for a.e. $x \in C[0, T]$. Suppose that $\int_{\mathbb{R}} \exp \{T|u|\} d \varphi(u)<\infty$ and $E\left[\exp \left\{\mid \int_{t_{j-1}}^{t_{j}} X(\cdot\right.\right.$, t)d $\left.\left.m_{L}(t) \mid\right\}\right]<\infty$ for $j=1, \ldots, n+1$. Then for $P_{Z_{n+1}}$ a.e. $\vec{\xi}_{n+1} \in$ $\mathbb{R}^{n+2} E\left[\exp \left\{\left|\int_{t_{j-1}}^{t_{j}} Z(\cdot, t) d m_{L}(t)\right|\right\} \mid Z_{n+1}\right]\left(\vec{\xi}_{n+1}\right)$ exists and

$$
\begin{aligned}
E & {\left[H_{Z} \mid Z_{n+1}\right]\left(\vec{\xi}_{n+1}\right) } \\
& =\prod_{j=1}^{n+1}\left[1+\sum_{m=1}^{\infty} \int_{\Delta_{m, j}} E\left[\left(H_{m}(\vec{s}, \cdot)\right)_{Z} \mid Z_{n+1}\right]\left(\vec{\xi}_{n+1}\right) d\left(m_{L}\right)^{m}(\vec{s})\right],
\end{aligned}
$$

where $\Delta_{m, j}=\left\{\left(s_{1}, \ldots, s_{m}\right): t_{j-1}<s_{1}<\cdots<s_{m}<t_{j}\right\}$ and $E\left[\left(H_{m}(\vec{s}, \cdot)\right)_{Z} \mid Z_{n+1}\right]$ is as given in Theorem 14. Moreover if $E\left[\exp \left\{\left|\int_{t_{j-1}}^{t_{j}} Z(\cdot, t) d m_{L}(t)\right|\right\} \mid Z_{n+1}\right](\overrightarrow{0})$ exists for $j=1, \ldots, n+$ 1, then $E\left[H_{Z} \mid Z_{n+1}\right]\left(\vec{\xi}_{n+1}\right)$ can be represented by

$$
\begin{aligned}
E & {\left[H_{Z} \mid Z_{n+1}\right]\left(\vec{\xi}_{n+1}\right) } \\
= & \exp \left\{\int_{0}^{T} P_{b, n+1}\left(\vec{\xi}_{n+1}\right)(t) d m_{L}(t)\right\} \\
& \times \prod_{j=1}^{n+1}\left[1+\sum_{m=1}^{\infty} \int_{\Delta_{m, j}} \int_{\mathbb{R}^{m}} u_{1} \cdots u_{m} \Psi_{m}(\vec{s}, \vec{u}) d\left(m_{L}\right)^{m}(\vec{u}) d\left(m_{L}\right)^{m}(\vec{s})\right] .
\end{aligned}
$$

Proof. By Theorem 2.1 and Lemma 2.1 of [6] we have for $j=$ $1, \ldots, n+1$

$$
\begin{aligned}
& E\left[\exp \left\{\left|\int_{t_{j-1}}^{t_{j}} Z(x, t) d m_{L}(t)\right|\right\}\right] \\
& \leq \int_{\mathbb{R}} \exp \{T|u|\} d \varphi(u) E\left[\exp \left\{\left|\int_{t_{j-1}}^{t_{j}} X(x, t) d m_{L}(t)\right|\right\}\right] \\
& \times \exp \left\{\left|\int_{t_{j-1}}^{t_{j}} a(t) d m_{L}(t)\right|\right\}<\infty
\end{aligned}
$$

so that $E\left[\exp \left\{\left|\int_{t_{j-1}}^{t_{j}} Z(\cdot, t) d m_{L}(t)\right|\right\} \mid Z_{n+1}\right]\left(\vec{\xi}_{n+1}\right)$ exists and

$$
\begin{aligned}
& E\left[\exp \left\{\int_{0}^{T} Z(x, t) d m_{L}(t)\right\}\right] \\
& \quad \leq E\left[\exp \left\{\sum_{j=1}^{n+1}\left|\int_{t_{j-1}}^{t_{j}} Z(x, t) d m_{L}(t)\right|\right\}\right]<\infty
\end{aligned}
$$

which implies the existence of $E\left[H_{Z} \mid Z_{n+1}\right]$. By the monotone convergence theorem we have

$$
\begin{aligned}
& \sum_{m=0}^{\infty} \frac{1}{m !} T^{m} \int_{\mathbb{R}}|u|^{m} d \varphi(u) \\
& \quad=\int_{\mathbb{R}} \exp \{T|u|\} d \varphi(u)<\infty
\end{aligned}
$$

so that we have $\int_{\mathbb{R}}|u|^{m} d \varphi(u)<\infty$ for each positive integer $m$. Furthermore for $P_{Z_{n+1}}$ a.e. $\vec{\xi}_{n+1} \in \mathbb{R}^{n+2}$ we have by Theorem 6

$$
\begin{aligned}
& \sum_{m=0}^{\infty} \frac{1}{m !} \int_{C[0, T]} \mid \int_{t_{j-1}}^{t_{j}}\left(Z_{b, n+1}(x, t)\right. \\
& \left.\quad+P_{b, n+1}\left(\vec{\xi}_{n+1}\right)(t)\right)\left.d m_{L}(t)\right|^{m} d w_{\varphi}(x) \\
& =\int_{C[0, T]} \exp \left\{\mid \int_{t_{j-1}}^{t_{j}}\left(Z_{b, n+1}(x, t)\right.\right. \\
& \left.\left.\quad+P_{b, n+1}\left(\vec{\xi}_{n+1}\right)(t)\right) d m_{L}(t) \mid\right\} d w_{\varphi}(x) \\
& =E\left[\exp \left\{\left|\int_{t_{j-1}}^{t_{j}} Z(x, t) d m_{L}(t)\right|\right\} \mid Z_{n+1}\right]\left(\vec{\xi}_{n+1}\right)<\infty .
\end{aligned}
$$


Now by Theorems 5, 6, 14, the Fubini's theorem, and the dominated convergence theorem we have for $P_{Z_{n+1}}$ a.e. $\vec{\xi}_{n+1} \in \mathbb{R}^{n+2}$

$$
\begin{gathered}
E\left[H_{Z} \mid Z_{n+1}\right]\left(\vec{\xi}_{n+1}\right) \\
=\int_{C[0, T]} \exp \left\{\int _ { 0 } ^ { T } \left(Z_{b, n+1}(x, t)\right.\right. \\
\left.\left.+P_{b, n+1}\left(\vec{\xi}_{n+1}\right)(t)\right) d m_{L}(t)\right\} d w_{\varphi}(x) \\
=\prod_{j=1}^{n+1}\left[\int _ { C [ 0 , T ] } \operatorname { e x p } \left\{\int _ { t _ { j - 1 } } ^ { t _ { j } } \left(Z_{b, n+1}(x, t)\right.\right.\right. \\
\left.\left.\left.+P_{b, n+1}\left(\vec{\xi}_{n+1}\right)(t)\right) d m_{L}(t)\right\} d w_{\varphi}(x)\right] \\
=\prod_{j=1}^{n+1}\left[1+\sum_{m=1}^{\infty} \int_{\Delta_{m, j}} E\left[\left(H_{m}(\vec{s}, \cdot)\right)_{Z} \mid Z_{n+1}\right]\left(\vec{\xi}_{n+1}\right) d\left(m_{L}\right)^{m}(\vec{s})\right]
\end{gathered}
$$

which proves the first equality of the theorem. Furthermore

$$
\begin{aligned}
E & {\left[H_{Z} \mid Z_{n+1}\right]\left(\vec{\xi}_{n+1}\right) } \\
= & \exp \left\{\int_{0}^{T} P_{b, n+1}\left(\vec{\xi}_{n+1}\right)(t) d m_{L}(t)\right\} \\
& \times\left[\prod_{j=1}^{n+1} \int_{C[0, T]} \exp \left\{\int_{t_{j-1}}^{t_{j}} Z_{b, n+1}(x, t) d m_{L}(t)\right\} d w_{\varphi}(x)\right] .
\end{aligned}
$$

Since (52) holds for $\vec{\xi}_{n+1}=\overrightarrow{0}$, we have by Theorem 13 and the dominated convergence theorem

$$
\begin{aligned}
& \int_{C[0, T]} \exp \left\{\int_{t_{j-1}}^{t_{j}} Z_{b, n+1}(x, t) d m_{L}(t)\right\} d w_{\varphi}(x) \\
& =1+\sum_{m=1}^{\infty} \int_{\Delta_{m, j}} \int_{C[0, T]} \prod_{l=1}^{m} Z_{b, n+1}\left(x, s_{l}\right) d w_{\varphi}(x) d\left(m_{L}\right)^{m}(\vec{s}) \\
& =1+\sum_{m=1}^{\infty} \int_{\Delta_{m, j}} \int_{\mathbb{R}^{m}} u_{1} \cdots u_{m} \Psi_{m}(\vec{s}, \vec{u}) d\left(m_{L}\right)^{m}(\vec{u}) d\left(m_{L}\right)^{m}(\vec{s})
\end{aligned}
$$

which proves the second equality of the theorem.

Theorem 21. Let the notations and the first part of the assumptions in Theorem 20 be given. Then for $P_{Z_{n}}$ a.e. $\vec{\xi}_{n} \in \mathbb{R}^{n+1}$ $E\left[H_{Z} \mid Z_{n}\right]\left(\vec{\xi}_{n}\right)$ is given by the first equality in Theorem 20 replacing $E\left[\left(H_{m}(\vec{s}, \cdot)\right)_{Z} \quad \mid \quad Z_{n+1}\right]\left(\vec{\xi}_{n+1}\right)$ by $E\left[\left(H_{m}(\vec{s}, \cdot)\right)_{Z} \quad \mid\right.$ $\left.Z_{n}\right]\left(\vec{\xi}_{n}\right)$, where the $E\left[\left(H_{m}(\vec{s}, \cdot)\right)_{Z} \mid Z_{n}\right]\left(\vec{\xi}_{n}\right)$ s are as given in Theorems 16 and 18. Moreover if the second part of the assumptions in Theorem 20 holds, then $E\left[H_{Z} \mid Z_{n}\right]\left(\vec{\xi}_{n}\right)$ is given by the second equality in Theorem 20 replacing $\exp \left\{\int_{0}^{T} P_{b, n+1}\left(\vec{\xi}_{n+1}\right)(t) d m_{L}(t)\right\}$ by

$$
\begin{aligned}
\exp \left\{\int_{0}^{t_{n}} P_{b, n}\left(\vec{\xi}_{n}\right)(t) d m_{L}(t)\right. \\
\quad+\frac{1}{2}\left(b(T)-b\left(t_{n}\right)\right)\left(B(T)-B\left(t_{n}\right)\right)^{2} \\
\left.\quad+\left(a(T)-a\left(t_{n}\right)\right)\left(B(T)-B\left(t_{n}\right)\right)+\xi_{n}\left(T-t_{n}\right)\right\},
\end{aligned}
$$

where $(d / d t) B(t)=\beta_{n+1}^{b}(t)=\left(b(t)-b\left(t_{n}\right)\right) /\left(b(T)-b\left(t_{n}\right)\right)$ and $\vec{\xi}_{n}=\left(\xi_{0}, \xi_{1}, \ldots, \xi_{n}\right)$.

Proof. The first part of the theorem immediately follows from Theorems 7, 16, 18, and 20. Suppose that the second part of the assumptions in Theorem 20 holds. For $\vec{\xi}_{n}=\left(\xi_{0}, \xi_{1}, \ldots, \xi_{n}\right) \in$ $\mathbb{R}^{n+1}$ let $\vec{\xi}_{n+1}=\left(\xi_{0}, \xi_{1}, \ldots, \xi_{n}, \xi_{n+1}\right)$, where $\xi_{n+1} \in \mathbb{R}$. Then we have

$$
\begin{aligned}
& {\left[\frac{1}{2 \pi\left(b(T)-b\left(t_{n}\right)\right)}\right]^{1 / 2}} \\
& \times \int_{\mathbb{R}} \exp \left\{\int_{0}^{T} P_{b, n+1}\left(\vec{\xi}_{n+1}\right)(t) d m_{L}(t)\right\} \\
& \times \exp \left\{-\left(\xi_{n+1}-a(T)-\left(\xi_{n}-a\left(t_{n}\right)\right)\right)^{2}\right. \\
& \left.\times\left(2\left(b(T)-b\left(t_{n}\right)\right)\right)^{-1}\right\} d m_{L}\left(\xi_{n+1}\right) \\
& =\left[\frac{1}{2 \pi\left(b(T)-b\left(t_{n}\right)\right)}\right]^{1 / 2} \\
& \times \int_{\mathbb{R}} \exp \left\{\int_{0}^{t_{n}} P_{b, n}\left(\vec{\xi}_{n}\right)(t) d m_{L}(t)\right. \\
& +\int_{t_{n}}^{T}\left(\beta_{n+1}(t)\left(\xi_{n+1}-\xi_{n}\right)+\xi_{n}\right) d m_{L}(t) \\
& -\left(\left(\xi_{n+1}-a(T)-\left(\xi_{n}-a\left(t_{n}\right)\right)\right)^{2}\right. \\
& \left.\left.\times\left(2\left(b(T)-b\left(t_{n}\right)\right)\right)^{-1}\right)\right\} d m_{L}\left(\xi_{n+1}\right) \\
& =\left[\frac{1}{2 \pi\left(b(T)-b\left(t_{n}\right)\right)}\right]^{1 / 2} \\
& \times \exp \left\{\int_{0}^{t_{n}} P_{b, n}\left(\vec{\xi}_{n}\right)(t) d m_{L}(t)+\left(T-t_{n}\right) \xi_{n}\right\} \\
& \times \int_{\mathbb{R}} \exp \left\{\left(a(T)-a\left(t_{n}\right)+u\right)\right. \\
& \times \int_{t_{n}}^{T} \beta_{n+1}^{b}(t) d m_{L}(t) \\
& \left.-\frac{u^{2}}{2\left(b(T)-b\left(t_{n}\right)\right)}\right\} d m_{L}(u)
\end{aligned}
$$




$$
\begin{aligned}
=\exp \left\{\int_{0}^{t_{n}} P_{b, n}\left(\vec{\xi}_{n}\right)(t) d m_{L}(t)+\xi_{n}\left(T-t_{n}\right)\right. \\
+\left(a(T)-a\left(t_{n}\right)\right)\left(B(T)-B\left(t_{n}\right)\right) \\
\left.+\frac{1}{2}\left(B(T)-B\left(t_{n}\right)\right)^{2}\left(b(T)-b\left(t_{n}\right)\right)\right\} .
\end{aligned}
$$

Now the second part of the theorem follows from Theorems 7 and 20.

Example 22. (1) Let $h \equiv 1$. Then $X(x, t)=x(t)-x(0)$ so that for $j=1, \ldots, n+1$

$$
\begin{aligned}
& E\left[\exp \left\{\left|\int_{t_{j-1}}^{t_{j}} X(\cdot, t) d m_{L}(t)\right|\right\}\right] \\
& \leq E\left[\exp \left\{T \sup _{0 \leq t \leq T}|x(t)-x(0)|\right\}\right]
\end{aligned}
$$

which is finite by Theorem 1.4 of [19]. Hence $E\left[H_{Z} \mid Z_{n+1}\right]$ and $E\left[H_{Z} \mid Z_{n}\right]$ are given by Theorems 20 and 21, respectively, with $b(t)=t$ and $(d / d t) B(t)=\left(t-t_{n}\right) /\left(T-t_{n}\right)$ for $t \in[0, T]$.

(2) Let $h(u)=T-u$ for $u \in[0, T]$ and suppose that $\int_{\mathbb{R}} \exp \{M|u|\} d \varphi(u)<\infty$, where $M=\max \left\{T, 2 T^{2}\right\}$. Then for $j=1, \ldots, n+1$ and $x \in C[0, T]$ we have by the integration parts formula

$$
\begin{aligned}
& \left|\int_{t_{j-1}}^{t_{j}} X(x, t) m_{L}(t)\right| \\
& =\mid \int_{t_{j-1}}^{t_{j}}[(T-t) x(t)-T x(0) \\
& \left.+\int_{0}^{t} x(s) d m_{L}(s)\right] d m_{L}(t) \\
& \leq \int_{t_{j-1}}^{t_{j}}\left[T \sup _{0 \leq s \leq T}|x(s)-x(0)|\right. \\
& +2 t \sup _{0 \leq s \leq T}|x(s)-x(0)| \\
& +2 t|x(0)|] d m_{L}(t) \\
& \leq 2 T^{2} \sup _{0 \leq s \leq T}|x(s)-x(0)|+T^{2}|x(0)|
\end{aligned}
$$

so that by Hölder's inequality

$$
\begin{aligned}
& \int_{C[0, T]} \exp \left\{\left|\int_{t_{j-1}}^{t_{j}} X(x, t) m_{L}(t)\right|\right\} d w_{\varphi}(x) \\
& \leq\left[\int_{\mathbb{R}} \exp \left\{2 T^{2}|u|\right\} d \varphi(u)\right]^{1 / 2} \\
& \quad \times\left[\int_{C[0, T]} \exp \left\{4 T^{2} \sup _{0 \leq s \leq T}|x(s)-x(0)|\right\} d w_{\varphi}(x)\right]^{1 / 2}
\end{aligned}
$$

which is finite by Theorem 1.4 of [19]. Now $E\left[H_{Z} \mid Z_{n+1}\right]$ and $E\left[H_{Z} \mid Z_{n}\right]$ are given by Theorems 20 and 21, respectively, with $b(t)=(1 / 3)\left[T^{3}-(T-t)^{3}\right]$ and $(d / d t) B(t)=1-((T-t) /$ $\left.\left(T-t_{n}\right)\right)^{3}$ for $t \in[0, T]$.

\section{Evaluation Formulas for Other Functions}

In this section, using the simple formulas in Section 2, we derive evaluation formulas for generalized conditional Wiener integrals of various functions which are of interest in Feynman integration theories themselves and quantum mechanics.

Since $Z_{b, n+1}(x, t)=Y(x, t)-P_{b, n+1}(Y(x, \cdot))(t)$ for $x \in$ $C[0, T]$ and $t \in[0, T]$ we have the following theorems from Theorem 3.2 of [11] and Theorems 21, 22, 23, 24, 25, and 26 of [10].

Theorem 23. Let $m \in \mathbb{N}$ and $F_{m}(x)=\int_{0}^{T}(x(t))^{m} d m_{L}(t)$ for $x \in C[0, T]$. Suppose that $\int_{\mathbb{R}}|u|^{m} d \varphi(u)<\infty$. Then for $P_{Z_{n+1}}$ a.e. $\vec{\xi}_{n+1} \in \mathbb{R}^{n+2}$

$$
\begin{aligned}
& E\left[\left(F_{m}\right)_{Z} \mid Z_{n+1}\right]\left(\vec{\xi}_{n+1}\right) \\
&=\sum_{j=1}^{n+1} \sum_{l=0}^{m / 2]} \frac{m !}{2^{l} l !(m-2 l) !} \int_{t_{j-1}}^{t_{j}}\left(A_{t}+P_{b, n+1}\left(\vec{\xi}_{n+1}\right)(t)\right)^{m-2 l} \\
& \times\left(\sigma_{j}^{b}(t, t)\right)^{l} d m_{L}(t)
\end{aligned}
$$

where $[m / 2]$ denotes the greatest integer less than or equal to $m / 2$.

Theorem 24. Let the assumptions be as given in Theorem 23 and for $\vec{\xi}_{n}=\left(\xi_{0}, \xi_{1}, \ldots, \xi_{n}\right) \in \mathbb{R}^{n+1}$ let

$$
\begin{aligned}
\Xi\left(\vec{\xi}_{n}\right)=\sum_{j=1}^{n} \sum_{l=0}^{[m / 2]} \frac{m !}{2^{l} l !(m-2 l) !} \int_{t_{j-1}}^{t_{j}} & \left(A_{t}+P_{b, n}\left(\vec{\xi}_{n}\right)(t)\right)^{m-2 l} \\
& \times\left(\sigma_{j}^{b}(t, t)\right)^{l} d m_{L}(t) .
\end{aligned}
$$

Then for $P_{Z_{n}}$ a.e. $\vec{\xi}_{n} \in \mathbb{R}^{n+1}$

$$
\begin{aligned}
& E\left[\left(F_{m}\right)_{Z} \mid Z_{n}\right]\left(\vec{\xi}_{n}\right) \\
& =\Xi\left(\vec{\xi}_{n}\right) \\
& +m ! \sum_{l=0}^{[m / 2] m-2 l} \sum_{k=0}^{k} \sum_{p=0}^{k} \sum_{q=0}^{[p / 2]}\left(\xi_{n}^{k-p}\left(a(T)-a\left(t_{n}\right)\right)^{p-2 q}\right. \\
& \left.\times\left(b(T)-b\left(t_{n}\right)\right)^{q}\right) \\
& \times\left(2^{l+q} l ! q !(k-p) !(p-2 q) !\right. \\
& \times(m-2 l-k) !)^{-1} \\
& \times \int_{t_{n}}^{T}\left(\sigma_{n+1}^{b}(t, t)\right)^{l}\left(\beta_{n+1}^{b}(t)\right)^{p} A_{t}^{m-2 l-k} d m_{L}(t) .
\end{aligned}
$$


Theorem 25. Let $0<s_{1}<s_{2} \leq T$ and let $s_{1} \in\left[t_{l-1}-t_{l}\right], s_{2} \in$ $\left[t_{j-1}-t_{j}\right]$ with $l \neq j$. For $x \in C[0, T]$ let $G(x)=x\left(s_{1}\right) x\left(s_{2}\right)$ and suppose that $\int_{\mathbb{R}} u^{2} d \varphi(u)<\infty$. Then for $P_{Z_{n+1}}$ a.e. $\vec{\xi}_{n+1} \in \mathbb{R}^{n+2}$

$$
\begin{aligned}
& E\left[G_{Z} \mid Z_{n+1}\right]\left(\vec{\xi}_{n+1}\right) \\
& \quad=\left(A_{s_{1}}+P_{b, n+1}\left(\vec{\xi}_{n+1}\right)\left(s_{1}\right)\right)\left(A_{s_{2}}+P_{b, n+1}\left(\vec{\xi}_{n+1}\right)\left(s_{2}\right)\right) .
\end{aligned}
$$

Theorem 26. Let the assumptions be as given in Theorem 25.

(1) If $l \leq n, j \leq n$, and $l \neq j$, then for $P_{Z_{n}}$ a.e. $\vec{\xi}_{n} \in \mathbb{R}^{n+1}$

$$
\begin{aligned}
E & {\left[G_{Z} \mid Z_{n}\right]\left(\vec{\xi}_{n}\right) } \\
& =\left(A_{s_{1}}+P_{b, n}\left(\vec{\xi}_{n}\right)\left(s_{1}\right)\right)\left(A_{s_{2}}+P_{b, n}\left(\vec{\xi}_{n}\right)\left(s_{2}\right)\right) .
\end{aligned}
$$

(2) If $l \leq n$ and $j=n+1$, then for $P_{Z_{n}}$ a.e. $\vec{\xi}_{n}=$ $\left(\xi_{0}, \xi_{1}, \ldots, \xi_{n}\right) \in \mathbb{R}^{n+1}$

$$
\begin{aligned}
E\left[G_{Z} \mid Z_{n}\right]\left(\vec{\xi}_{n}\right) & \\
= & \left(A_{s_{2}}+\xi_{n}+\beta_{n+1}^{b}\left(s_{2}\right)\left(a(T)-a\left(t_{n}\right)\right)\right) \\
& \times\left(A_{s_{1}}+P_{b, n}\left(\vec{\xi}_{n}\right)\left(s_{1}\right)\right) .
\end{aligned}
$$

For $j=1, \ldots, n+1$, let $g_{j}=\left(1 / \sqrt{b\left(t_{j}\right)-b\left(t_{j-1}\right)}\right) \chi_{\left(t_{j-1}, t_{j}\right]} h$, let $V$ be the subspace of $L_{2}[0, T]$ generated by $\left\{g_{1}, \ldots, g_{n+1}\right\}$, let $V^{\perp}$ be the orthogonal complement of $V$ and let $\mathscr{P}^{\perp}$ : $L_{2}[0, T] \rightarrow V^{\perp}$ be the orthogonal projection. Let $\mathscr{M}\left(L_{2}[0, T]\right)$ be the class of all complex valued Borel measures of bounded variation over $L_{2}[0, T]$ and let $\mathcal{S}_{w_{\varphi}}$ be the space of all functions $F$ which for $\sigma \in \mathscr{M}\left(L_{2}[0, T]\right)$ have the form

$$
F(x)=\int_{L_{2}[0, T]} \exp \{i(v, x)\} d \sigma(v)
$$

for $w_{\varphi}$ a.e. $x \in C[0, T]$. Note that $\mathcal{S}_{w_{\varphi}}$ is a Banach algebra [6].

Theorem 27. Let a be absolutely continuous on $[0, T]$. Let $F \in$ $\mathcal{S}_{w_{\varphi}}$ and $\sigma \in \mathscr{M}\left(L_{2}[0, T]\right)$ be related by (67). Then for $P_{Z_{n+1}}$ a.e. $\vec{\xi}_{n+1} \in \mathbb{R}^{n+2}, E\left[F_{Z} \mid Z_{n+1}\right]\left(\vec{\xi}_{n+1}\right)$ is given by

$$
\begin{aligned}
& E\left[F_{Z} \mid Z_{n+1}\right]\left(\vec{\xi}_{n+1}\right) \\
& =\int_{L_{2}[0, T]} \exp \left\{i\left(v, A_{t}+P_{b, n+1}\left(\vec{\xi}_{n+1}\right)\right)\right\} \\
& \quad \times \exp \left\{-\frac{1}{2}\left\|\mathscr{P}^{\perp}(v h)\right\|_{2}^{2}\right\} d \sigma(v) .
\end{aligned}
$$

Theorem 28. Let the assumptions be as given in Theorem 27 and for $\vec{\xi}_{n} \in \mathbb{R}^{n+1}$ let

$$
D\left(v, \vec{\xi}_{n}\right)=\exp \left\{i\left(v, A_{t}+P_{b, n}\left(\vec{\xi}_{n}\right)\right)-\frac{1}{2}\left\|\mathscr{P}^{\perp}(v h)\right\|_{2}^{2}\right\} .
$$

Then for $P_{Z_{n}}$ a.e. $\vec{\xi}_{n} \in \mathbb{R}^{n+1}, E\left[F_{Z} \mid Z_{n}\right]\left(\vec{\xi}_{n}\right)$ is given by

$$
\begin{aligned}
& E\left[F_{Z} \mid Z_{n}\right]\left(\vec{\xi}_{n}\right) \\
& =\int_{L_{2}[0, T]} D\left(v, \vec{\xi}_{n}\right) \exp \left\{i \frac{\left\langle v h, g_{n+1}\right\rangle_{2}\left(a(T)-a\left(t_{n}\right)\right)}{\sqrt{b(T)-b\left(t_{n}\right)}}\right. \\
& \left.-\frac{1}{2}\left\langle v h, g_{n+1}\right\rangle_{2}^{2}\right\} d \sigma(v) \text {. }
\end{aligned}
$$

Remark 29. Suppose that $\int_{\mathbb{R}} u^{2} d \varphi(u)<\infty$, if necessary, in each lemma, theorem, and example of this paper. Then by Lemma 2.5 of [11] both $E\left[X_{0}\right]$ and $\operatorname{Var}\left[X_{0}\right]$ exist. Let $m_{Z}(t)=$ $a(t)+E\left[X_{0}\right]$ and $b_{Z}(t)=b(t)+\operatorname{Var}\left[X_{0}\right]$ for $t \in[0, T]$. Since for $t_{1}, t_{2} \in[0, T]$

$$
\begin{gathered}
m_{Z}\left(t_{2}\right)-m_{Z}\left(t_{1}\right)=a\left(t_{2}\right)-a\left(t_{1}\right), \\
b_{Z}\left(t_{2}\right)-b_{Z}\left(t_{1}\right)=b\left(t_{2}\right)-b\left(t_{1}\right),
\end{gathered}
$$

$a$ and $b$ can be replaced by $m_{Z}$ and $b_{Z}$, respectively, in each result of this paper.

\section{Conflict of Interests}

The author declares that there is no conflict of interests regarding the publication of this paper.

\section{Acknowledgments}

This research was supported by Basic Science Research Program through the National Research Foundation of Korea (NRF) funded by the Ministry of Education (2013R1A1A2058991).

\section{References}

[1] I. Pierce, On a family of generalized Wiener spaces and applications [Ph.D. thesis], University of Nebraska-Lincoln, Lincoln, Neb, USA, 2011.

[2] G. W. Johnson and M. L. Lapidus, The Feynman Integral and Feynman's Operational Calculus, Oxford Mathematical Monographs, Oxford University Press, The Clarendon Press, New York, NY, USA, 2000.

[3] R. H. Cameron and D. A. Storvick, "Some Banach algebras of analytic Feynman integrable functionals," in Analytic Functions Kozubnik 1979, vol. 798 of Lecture Notes in Mathematics, pp. 1867, Springer, Berlin, Germany, 1980.

[4] G. W. Johnson and D. L. Skoug, "Notes on the Feynman integral. I," Pacific Journal of Mathematics, vol. 93, no. 2, pp. 313-324, 1981.

[5] C. Park and D. Skoug, "A Kac-Feynman integral equation for conditional Wiener integrals," Journal of Integral Equations and Applications, vol. 3, no. 3, pp. 411-427, 1991.

[6] M. K. Im and K. S. Ryu, "An analogue of Wiener measure and its applications," Journal of the Korean Mathematical Society, vol. 39 , no. 5, pp. 801-819, 2002. 
[7] K. S. Ryu and M. K. Im, "A measure-valued analogue of Wiener measure and the measure-valued Feynman-Kac formula," Transactions of the American Mathematical Society, vol. 354, no. 12, pp. 4921-4951, 2002.

[8] K. S. Ryu, M. K. Im, and K. S. Choi, "Survey of the theories for analogue of Wiener measure space," Interdisciplinary Information Sciences, vol. 15, no. 3, pp. 319-337, 2009.

[9] D. H. Cho, "A simple formula for a generalized conditional Wiener integral and its applications," International Journal of Mathematical Analysis, vol. 7, no. 29-32, pp. 1419-1431, 2013.

[10] D. H. Cho, "Evaluation formulas for generalized conditional Wiener integrals with drift on a function space," Journal of Function Spaces and Applications, vol. 2013, Article ID 469840, 9 pages, 2013.

[11] D. H. Cho, "Evaluation formulas for generalized Wiener integrals with drift and initial distribution," International Journal of Mathematical Analysis, vol. 8, no. 2, pp. 51-65, 2014.

[12] S. J. Chang and D. M. Chung, "Conditional function space integrals with applications," The Rocky Mountain Journal of Mathematics, vol. 26, no. 1, pp. 37-62, 1996.

[13] D. H. Cho, "A simple formula for an analogue of conditional Wiener integrals and its applications," Transactions of the American Mathematical Society, vol. 360, no. 7, pp. 3795-3811, 2008.

[14] D. H. Cho, "A simple formula for an analogue of conditional Wiener integrals and its applications. II," Czechoslovak Mathematical Journal, vol. 59, no. 2, pp. 431-452, 2009.

[15] C. Park and D. Skoug, "A simple formula for conditional Wiener integrals with applications," Pacific Journal of Mathematics, vol. 135, no. 2, pp. 381-394, 1988.

[16] R. B. Ash, Real Analysis and Probability, Probability and Mathematical Statistics, no. 11, Academic Press, New York, NY, USA, 1972.

[17] S. J. Chang and D. Skoug, "The effect of drift on conditional Fourier-Feynman transforms and conditional convolution products," International Journal of Applied Mathematics, vol. 2, no. 4, pp. 505-527, 2000.

[18] S. S. Wilks, Mathematical Statistics, A Wiley Publication in Mathematical Statistics, John Wiley \& Sons, New York, NY, USA, 1962.

[19] Y. H. Park, "Evaluation $E\left(\exp \left(\int_{0}^{t} h(s) \widetilde{d} x(s)\right)\right)$ on analogue of Wiener measure space," Honam Mathematical Journal, vol. 32, no. 3, pp. 441-451, 2010. 


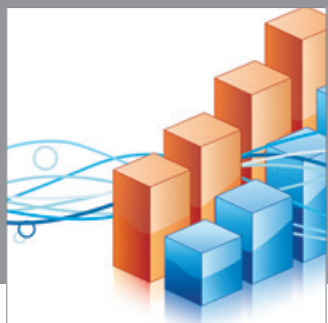

Advances in

Operations Research

mansans

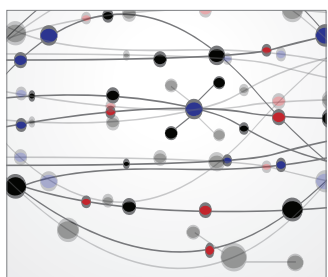

The Scientific World Journal
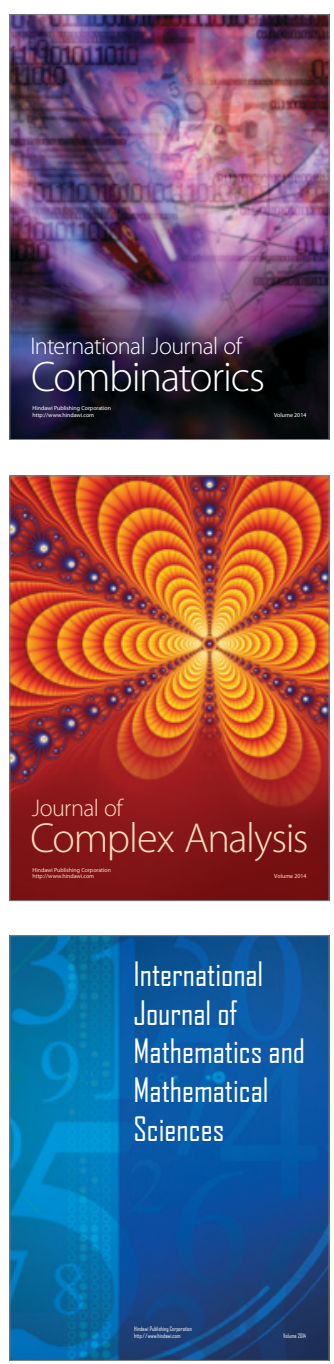
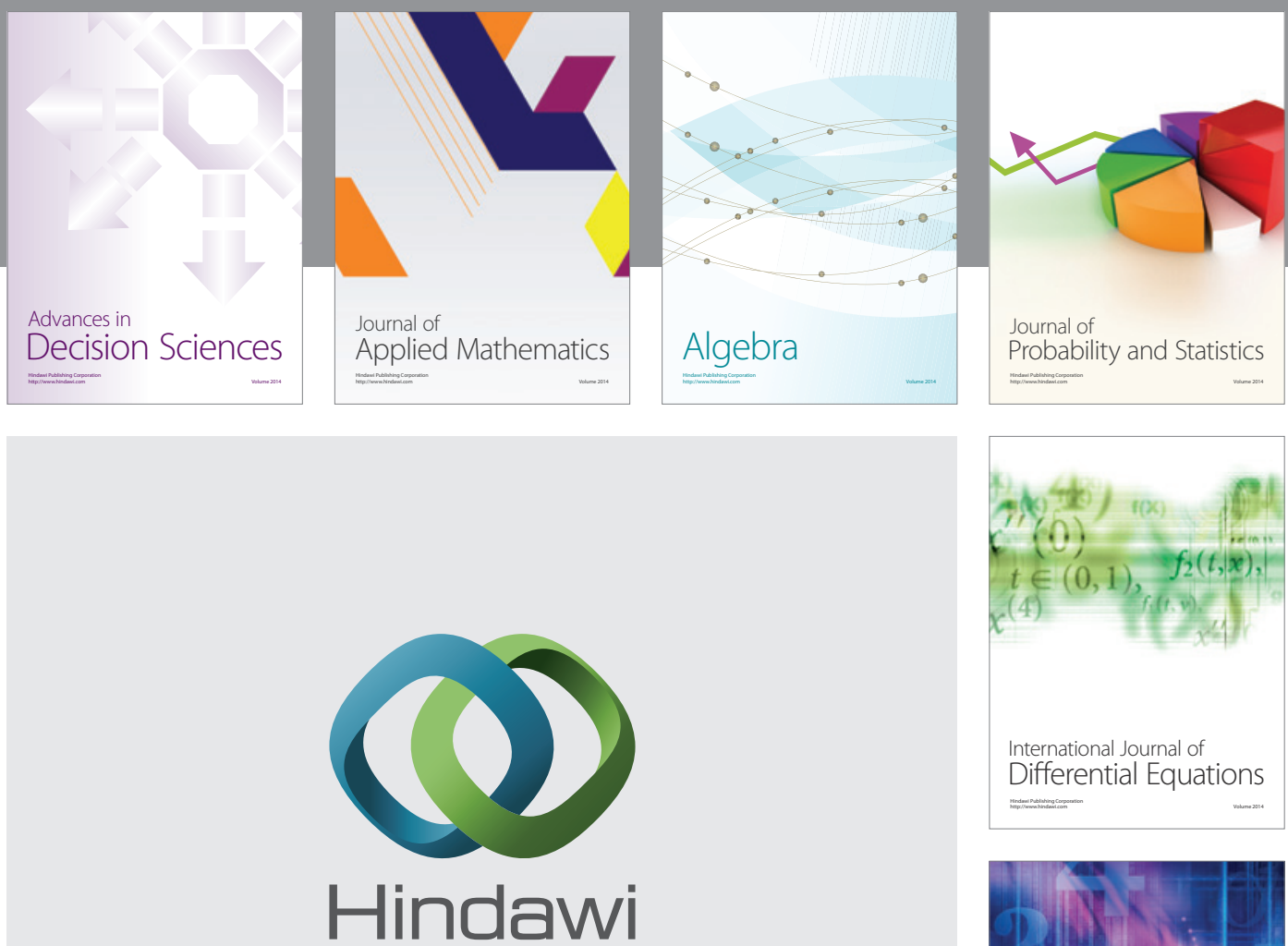

Submit your manuscripts at http://www.hindawi.com
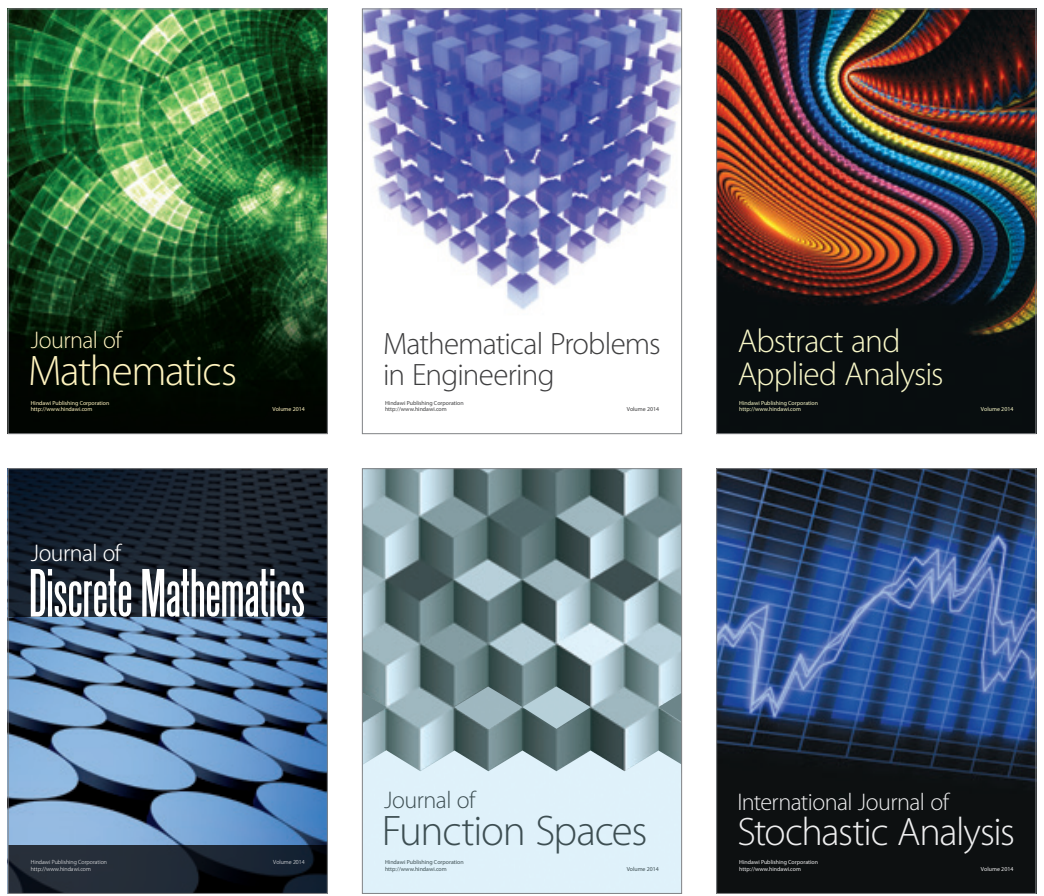

Journal of

Function Spaces

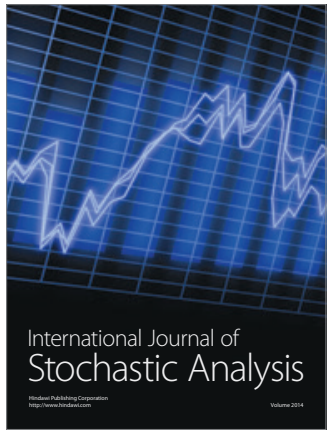

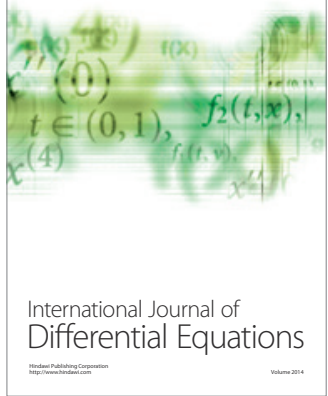
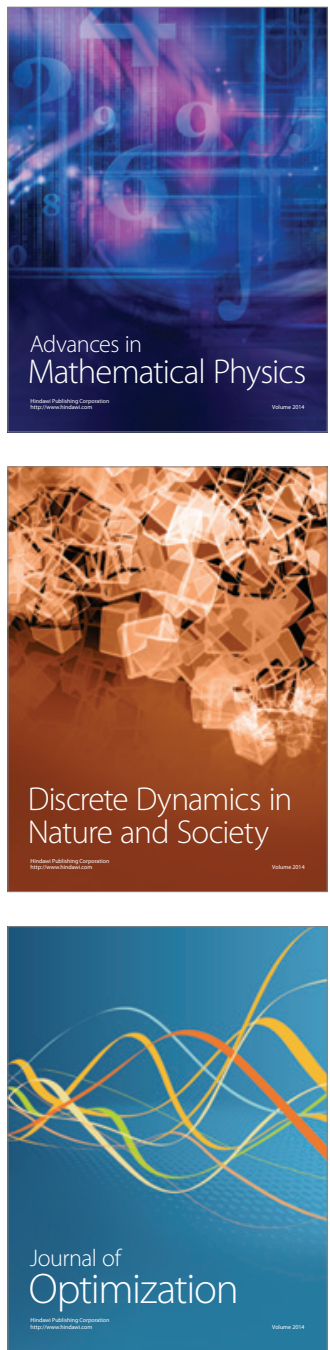